3

4

5

6

7

9

10

11

12

13

14

15 Corresponding author: Joshua Penalba (josh.penalba@gmail.com)

16

17

18

19

20

21

22

23

24

25

26

27

28

29

30

31

32

33

34 northern Australian birds

\author{
Joshua V. Peñalba ${ }^{1,2,3}$, Leo Joseph ${ }^{2,3}$, Craig Moritz ${ }^{1,2}$
}

\begin{abstract}
Author affiliations
\end{abstract}
${ }^{2}$ Centre for Biodiversity Analysis, Acton, ACT 2601, Australia Canberra, GPO BOX 1700, Canberra, ACT 2601, Australia

6

Key words: geographic mode, allopatry, parapatry, snowballing, tipping point

8

9

1

3

(5)

6

7

(2)

0

2

4

\title{
Current geography masks dynamic history of gene flow during speciation in
}

${ }^{1}$ Ecology \& Evolution, Australian National University, Acton, ACT 2601, Australia

${ }^{3}$ Australian National Wildlife Collection, CSIRO National Research Collections Australia, 


\section{Abstract}

During early stages of speciation, genome divergence is greatly influenced by gene flow.

37 As populations diverge, geography can allow or restrict gene flow in the form of barriers.

38 Current geography, e.g. whether sister species are allopatric or parapatric, is often used to predict

39 the potential for gene flow during the divergence process. We test the validity of this assumption

40 in eight meliphagoid bird species codistributed across four regions. These regions are separated

41 by known biogeographic barriers within and between northern Australia and Papua New Guinea.

42 We find that bird populations across the same barrier have a range of divergence levels and

43 probability of gene flow regardless of range connectivity. Geographic distance and maximum

44 range connectivity over time can better predict divergence and probability of gene flow than

45 whether populations are currently allopatric or parapatric. We also find support for a nonlinear

46 decrease of the probability of gene flow during the divergence process. This implies that

47 although gene flow influences divergence early in speciation, other factors associated with

48 higher divergence restrict gene flow later in speciation. Current geography may then mislead

49 inferences regarding potential for gene flow during speciation under a complex and dynamic

50 history of geographic and reproductive isolation.

\section{Background}

52 Gene flow, selection, and genetic drift shape divergence during speciation, while

53 geography sets the stage on which these forces act [1-3]. The geographic mode of speciation is

54 defined by the extent of spatial isolation during early stages of divergence. Population pairs can

55 have disjoint (allopatric), completely overlapping (sympatric), or separate yet partially adjoining

56 (parapatric) ranges [4]. This geographic context predicts potential gene flow between

57 populations. Variation in levels of gene flow affects genetic differentiation, in turn affecting the 
58 strength of selection and drift that is necessary to drive population divergence. Alternatively,

59 under a purely population genetic framework the geographic mode of speciation is defined by

60 levels of gene flow; "allopatry" when the proportion of the population which are migrants $(\mathrm{m})$

61 equals zero, "sympatry" when $m=0.5$, and "parapatry" when $0<m<0.5$ per generation [5].

Although geographic and genetic definitions are often assumed to correspond, this is not

63 always the case in nature [6]. Current range distributions between sister species are often used to

64 infer their geographic mode of speciation, although the dynamic nature of species ranges through

65 evolutionary time can lead to unreliable predictions of gene flow [7]. Range fluctuations during

66 the Pleistocene's climatic cycling resulted in multiple periods of connectivity and discontinuity

67 before resulting in the distribution we see today [8]. In order to understand how gene flow has

68 influenced divergence during speciation, we must first understand how the geographic history

69 could have shaped the potential for gene flow through time. The discrepancy between the spatial

70 and population genetic definitions of the geographic mode begs the question: does current

71 geography adequately predict realized gene flow and, consequently, divergence during early

72 stages of speciation?

73 Although geographic connectivity would allow for gene flow early in speciation, later in

74 the proces geographic connectivity can be insufficient for gene flow as populations diverge and

75 become reproductively isolated. Populations further along in the speciation process would have

76 reduced realized gene flow due to intrinsic incompatibilities or extrinsic selection [9]. Especially

77 in birds, prezygotic isolation from sexual selection on song or plumage could influence gene

78 flow in later stages of speciation. There has been growing support for the "snowball" model of

79 accumulating incompatibility loci, initially proposed by Orr [10]. Qualitatively, this model and

80 those derived from it specify a nonlinear accumulation of incompatibility loci resulting in a short 
81 speciation duration [11]. The "snowballing" has also been modeled under scenarios with

82 moderate to no gene flow (parapatry to allopatry) meaning different geographic modes may yield

83 a similar short duration of speciation, varying only in how long it takes for speciation to initiate

84 [12-16]. This rapid accumulation of isolation has also been proposed under models of divergent

85 selection in speciation-with-gene flow and under neutral models [17-20]. Though the underlying

86 assumptions of these theories may differ, the trajectory converges to a rapid transition,

87 'snowballing', or 'tipping point' during speciation (simply referred to 'snowballing' from this

88 point forward). There is increasing empirical support for this pattern from studies of individual

89 systems [21-23] and a taxonomically broad meta-analysis [24]. More broad, comparative studies

90 across multiple systems would help elucidate this trajectory to speciation.

91 Further studies of population divergence in various geographic contexts could further

92 clarify the role of gene flow, selection and genome architecture in influencing the landscape of

93 divergence $[9,20,25,26]$. During speciation, local genomic variation in mutation and

94 recombination rates influence the rate at which regions diverge $[25,27,28]$. The geographic mode

95 of speciation could influence this landscape of divergence. Theory predicts that populations

96 diverging in parapatry should have a skewed distribution of divergence across the genome with a

97 few loci resistant to gene flow due to selection while the rest are free to move between

98 populations [29]. Populations diverging in allopatry, on the other hand, are predicted to lack this

99 skew as drift would be the predominant force influencing divergence [30,31]. This landscape

100 should also change as populations move further along the speciation continuum $[9,27]$.

101 Here we investigate multiple bird species with populations codistributed in the same

102 region with known biogegraphic barriers. Empirical studies of gene flow during divergence in

103 relation to geography often survey closely related populations and species across different 
geographic regions with varying biogeographic histories and selection pressures [30,31].

105

106

107

108

109 between closely related systems.

\section{Methods}

However, to understand how shifting geographic ranges can erode the correspondence between current geography and realized gene flow, it is more relevant to compare a set of taxa across common geography. Our study region comprises part of the monsoonal tropics of northern Australia and southern Papua New Guinea containing congruent biogeographic barriers for many taxa including birds [32-34]. Sea level rise since the last glacial maximum has formed a barrier between northern Australia and Papua New Guinea [35]. Meanwhile, the aridification of mainland Australia has resulted in multiple semipermeable terrestrial barriers with parapatrically or allopatrically distributed populations. Multiple studies have shown congruent phenotypic and genetic breaks for various plant and animal species in this region [34,36-39]. Here we use one gerygone and seven honeyeater species co-distributed in four focal regions: Papua New Guinea (PNG), Northern Territory (NT), Cape York Peninsula (CYP) and eastern Queensland (QLD) that are separated by well-known barriers (figure 1). These species were chosen as they have already been shown to have variation in divergence levels across known barriers between CYP and QLD [40] and have varying degrees of range connectivity [41] setting the stage to compare divergence in different biogeographical contexts. With this system we ask (1) how current geography, or potential for gene flow, predicts realized gene flow and genome divergence during early stages of speciation, (2) how genome divergence influences realized gene flow in later stages of speciation, (3) and how divergence varies across different loci during the speciation process. Focusing on the codistributed ranges of these species will allow us to understand how the genome differentiates during speciation under different geographic contexts within and 
Sampling

We sampled eight species with populations that occupy the four regions of interest

129 (figure 1). For each population, we sampled three to six individuals for a total of 157 individuals

130 (15-20 individuals per species; electronic supplementary material, table S1). Samples were

131 chosen from locations farther away from known contact zones to avoid recent hybrids [42].

132 Brown honeyeater and white-throated honeyeater had insufficient sampling for PNG so only the 133 Australian populations were used in the analyses. We extracted the DNA from all individuals

134 using a standard salting-out procedure.

135 Sequencing

We sanger sequenced NADH dehydrogenase-2 (ND2) using the primers L5204 (5'

137 TAACTAAGCTATCGGGCGCAT 3') and H6312 (5'CTTATTTAAGGCTTTGAAGGCC 3')

138 for measures of mitochondrial divergence and structure [43]. For the nuclear loci, we used a

139 slightly modified version of the ddRADseq protocol as described by [44]. In brief, we digested

140 the DNA using the restriction enzymes PstI and EcoRI and size-selected 345 - 407bp.

141 Approximately ten indexed individuals in ten pools were sequenced on a NextSeq500 for 150bp,

142 single-read, mid-output and the rest were sequenced on another NextSeq500 lane with similar

143 specification except with high-output. A more detailed description of lab methods is available in

144 the electronic supplementary material.

145 Data processing and analyses

146 For each species we generated a reference set of RAD loci via the pyrad pipeline [45].

147 We used individuals from a different species to serve as an outgroup to polarize the SNPs in

148 downstream analyses. Only RAD loci which have associated outgroup sequences were retained

149 for the reference (electronic supplementary material, table S2). The resulting set of sequences 
150 were used as a reference for further processing. Individual reads were then mapped onto this

151 reference set using Bowtie2 (v. 2.2.2)[46]. In order to recover larger numbers of loci and avoid

152 biases from filtering for loci with higher coverage, the mapped reads were further processed

153 using ngsTools and ANGSD to incorporate genotype likelihood information for the various

154 population genetic measures (v. 0.911; http://www.popgen.dk/angsd) [47].

155 Population structure

It has been demonstrated that more loci, even of lower coverage, yields more accurate

157 estimates of population genetic statistics compared to fewer loci of higher coverage when using

158 genotype likelihoods [48].We used ANGSD to further filter for SNPs to be used for downstream

159 analyses (electronic supplementary material, table S3; see electronic supplementary material for

160 detailed ANGSD commands). We used a minimum coverage cut-off of $2 \mathrm{X}$ and a maximum cut-

161 off of 40X per individual to optimize the number of loci to be used while reducing the likelihood

162 of recovering paralogous loci. The maximum coverage cut-off was determined by plotting a

163 histogram of the average coverage per RAD locus and finding the upper threshold where most

164 loci fell under. To determine population structure, we randomly chose a single SNP per RAD

165 locus and reran ANGSD only for that set. We then used used ngsDist [49] to generate a distance

166 matrix which we used for a principal coordinates analysis (PCoA) using 'cmdscale' from base $\mathrm{R}$

167 (v3.2.2) and a population network using SplitsTree (figure 1, electronic supplementary material

168 figure S1) [50].

169 Population divergence statistics

170 To calculate the various population divergence statistics $\left(\mathrm{F}_{\mathrm{ST}}, \mathrm{D}_{\mathrm{XY}}\right.$, and $\left.\mathrm{D}_{\mathrm{A}}\right)$ we used the

171 software within the ngsTools package. These statistics were used as they provide both relative

172 and absolute measures of genetic divergence and can be compared to previous studies [24,30,31]. 
173 We used all SNPs within each RAD locus for all per locus and global measures. To calculate

174 pairwise $\mathrm{F}_{\mathrm{ST}}$, we used realSFS on the ANGSD genotype likelihoods to estimate an unfolded 2D

175 site frequency spectrum (SFS) per population pair and used the SFS to derive the per locus $\mathrm{F}_{\mathrm{ST}}$

176 estimate [51,52]. We used the same outgroups as the pyrad filtering steps for the unfolded SFS.

177 To calculate global $\mathrm{F}_{\mathrm{ST}}$, we used the estimate of allele frequencies within each population and all

178 populations pooled together $\mathrm{F}_{\mathrm{ST}}=\mathrm{H}_{\mathrm{T}}-\mathrm{H}_{\mathrm{S}} / \mathrm{H}_{\mathrm{T}}$ where $\mathrm{H}_{\mathrm{T}}$ is the heterozygosity of all populations

179 pooled and $H_{S}=\Sigma H_{e} / k$ and $H_{e}=1-\Sigma\left(p^{2}+(1-p)^{2}\right) / m$ where $\mathrm{k}$ is the number of populations, $m$

180 is the number of loci, and $\mathrm{p}$ is the allele frequency [53]. To calculate $\mathrm{D}_{\mathrm{XY}}$, we used the estimate

181 of allele frequencies which incorporated the genotype likelihood from ANGSD [54]. To

182 calculate $\mathrm{D}_{\mathrm{A}}$, we used the $\pi$ estimates from $\mathrm{ANGSD}$ for each population and the equation $\mathrm{D}_{\mathrm{A}}=$

$183 \mathrm{D}_{\mathrm{XY}}-\left(\pi_{\mathrm{X}}+\pi_{\mathrm{Y}}\right) / 2$. Lastly, we used the package ape v. 4.1 to calculate the ND2 genetic p-distance

184 under the Jukes-Cantor model [55].

185 Estimating the likelihood of migration

186 We used an approximate Bayesian computation (ABC) model selection to estimate the

187 likelihood of migration (i.e. how strong the evidence is for some gene flow during the divergence

188 process) between each population pair. The ABC analyses and models followed that of Roux et

189 al. but using the unfolded 2DSFS as a summary statistic instead of the various population genetic

190 statistics used in their study [24]. We tested the models of isolation-with-migration (IM),

191 isolation-with-migration + heterogeneous $\mathrm{Ne}(\mathrm{IMhetN})$, isolation-with-migration +

192 heterogeneous migration (IMhetM), isolation-with-migration + heterogeneous $\mathrm{Ne}+$

193 heterogeneous migration (IMhetNhetM), strict isolation (SI), and strict isolation + heterogeneous

$194 \mathrm{Ne}$ (SIhetN). Heterogeneous $\mathrm{Ne}$ is modeled to reflect variation in recombination rate throughout

195 the genome and heterogeneous migration is to reflect variation in gene flow across loci between 
hybridizing populations. Models of SI preceding IM (secondary contact) and IM preceding SI were excluded as they are typically difficult to distinguish from IM models [24]. We then used 50 trained and 6 hidden networks [56]. We ran the abc analyses five times and used the average model support of the replicates for further analyses. As in Roux et al., we used the sum of the

201 support for models containing a migration parameter as our probability for migration [24].

202 Additional details of the analyses can be found in the supplementary material.

To infer the relationship between divergence and realized gene flow, we correlated $\mathrm{F}_{\mathrm{ST}}$ to

204 the probability for migration between each population pair. To negate possible circularity where model support may be determined by $\mathrm{F}_{\mathrm{ST}}$ itself, we calculated the $\mathrm{F}_{\mathrm{ST}}$ of simulations under the SI model and reran the $\mathrm{ABC}$ model selection on a range of $\mathrm{F}_{\mathrm{ST}}$ values. If the $\mathrm{ABC}$ model selection

207 consistently recovers a low probability of migration, regardless of $\mathrm{F}_{\mathrm{ST}}$, high support for IM for

208 populations with lower $\mathrm{F}_{\mathrm{ST}}$ in the empirical data would more likely be a biological phenomenon 209 rather than a model selection artifact.

\section{Speciation model fitting}

211 We fit our divergence versus probability of migration distribution to test support for

212 various theoretical trajectories of parapatric speciation presented by Yamaguchi and Iwasa [15].

213 The models include (1) 'threshold': where full incompatibility is reached after a certain

214 divergence level, (2) 'constant' rate of divergence increase, (3) 'accelerated' where increase in

215 divergence is small until a certain divergence threshold is reached and increase is accelerated, (4)

216 'decelerated' increase where divergence accumulates quickly but slows down as it approaches

217 full incompatibility, and (5) 'sigmoid' where the rate of increase starts slow but accelerates after

218 a certain threshold before decelerating again prior to reaching complete incompatibility 
219 suggesting a snowballing during the speciation process (electronic supplementary material, table

220 S4, figure S2). Our global $F_{\text {ST }}$ parallels the 'incompatibility genetic distance' of Yamaguchi and

221 Iwasa [15]. The inverse of our probability of migration parallels their 'incompatibility' where no

222 support for migration $(P(m)=0))$ is equivalent to one or full support for incompatibility $(I(\mathrm{z})=$

223 1). Identical to $\mathrm{F}_{\mathrm{ST}}$ and probability of migration, the measures Yamaguchi and Iwasa used are

224 bounded by zero and one. We used the resulting probability of migration as the summary statistic

225 and simulated 500k distributions under each speciation trajectory model and used ABC and

226 model rejection to estimate model support for our data. To test robustness of our ABC model

227 inference, we used 200 simulated datasets under each model to see if the correct model is

228 recovered (electronic supplementary material, table S5). Details regarding this method can be

229 found in the supplementary material.

$230 \quad$ Species distribution modeling

231 To infer how geographic distributions have changed through time we estimated

232 geographic connectivity over space through time using species distribution modeling and least

233 cost path analyses. We used vouchered specimen data from the Atlas of Living Australia

234 (http://www.ala.org.au) for occurrence points and environmental variables from WorldClim v.

2351.4 (Community Climate System Model 4; http://worldclim.org/paleo-climate1) to predict

236 species ranges under past climates. To model species distributions in R v. 3.2.2, we followed

237 guidelines described in [57]. We used the R package dismo v. 1.1-1 for the maximum entropy

238 (MAXENT) analyses to predict species ranges [57]. We ran MAXENT using environmental

239 layers from the present, mid-Holocene and the LGM (electronic supplementary material, table

240 S6). Lastly, we selected a single coordinate (midpoint of the range) for each of the four

241 populations and calculated the geographic distance between those points to account for isolation- 
242 by-distance. Between population pairs, we also calculated the least cost path using the R package

243 gdistance (v. 1.1-9) based on modeled suitability [58] in the current range, mid-Holocene, and

244 LGM predictions. For each population pair, we chose the minimum resistance path between all

245 three time points to quantify the highest opportunity for migration through time. Habitat

246 resistance values can be found in the electronic supplementary material table S7 and species

247 distribution predictions can be found in figure S3.

248 Results

249 Population structure and divergence

250 Population structure varied between each taxon-pair though most had some form of

251 clustering across the four populations (figure 1). Of the eight species, five showed distinct

252 clusters for all four populations, one showed only three distinct clusters, one showed only two

253 distinct clusters, and the last showed no population clusters ( $\left.\mathrm{F}_{\mathrm{ST}} 0.09-0.39\right)$. The degrees of

254 clustering and the population relationships are fairly variable, as shown by a PCoA and

255 population network of the genetic distances (electronic supplementary material, figure S1).

256 Currently allopatric populations are not more likely to be separate clusters, exemplified by

257 samples from the PNG population often being closer to CYP and QLD. Similarly, geographically

258 parapatric populations are not necessarily mixed as as shown by NT often being the most

259 diverged and consistently separate cluster even for species with currently continuous geographic

260 ranges to CYP and QLD. Mitochondrial haplotype networks generally corroborated nuclear

261 SNP structure. Measures of genetic differentiation and divergence also varied. Autosomal and Z

262 chromosome divergences were consistently correlated with all divergence measures (electronic

263 supplementary material, figure $\mathrm{S} 4$, tables S8 and S9). Relative divergence $\left(\mathrm{F}_{\mathrm{ST}}\right)$ and gene flow

264 scales with mitochondrial divergence with a few outliers. The transition to low probability of 
migration is fairly rapid beyond $1 \%$ ND2 p-distance. $\mathrm{D}_{\mathrm{XY}}$, an absolute measure of divergence, also scales with $\mathrm{F}_{\mathrm{ST}}$ but with more outliers compared to ND2 (electronic supplementary material,

267 figure S5). Though different measures of divergence in different DNA classes generally

268 correlated as expected, divergence and structure of populations varied between species with no

269 immediate patterns corresponding to geography.
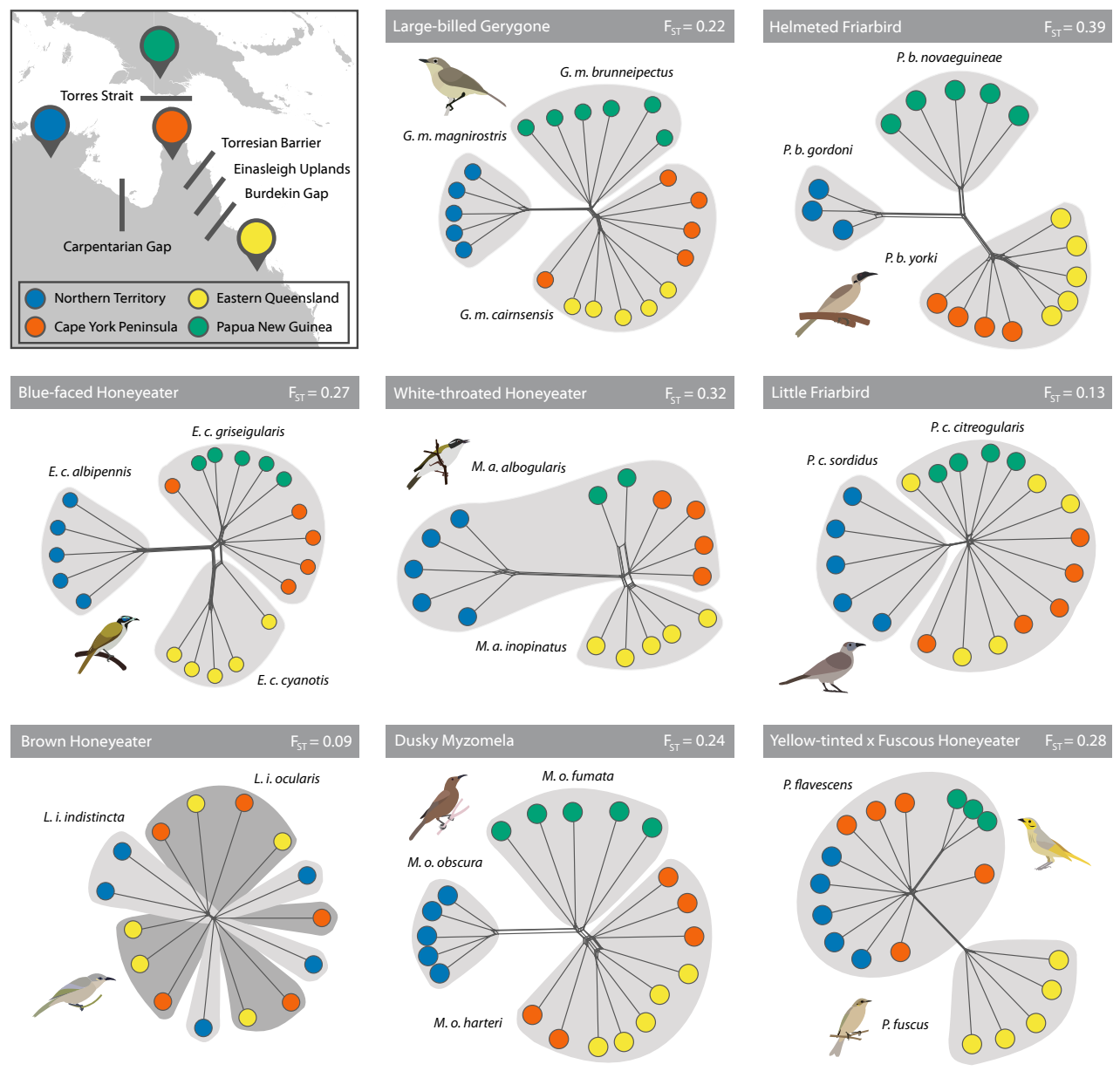

271 Figure 1. Geographic regions that were sampled for this study with corresponding known

272 biogeographical barriers. Sample networks were generated from a distance matrix estimated

273 from the ddRAD genotype likelihoods. Within each system, the species and subspecies

274 designation has been highlighted. $\mathrm{F}_{\mathrm{ST}}$ represents global measures across all four populations. 


\section{Geography and genome divergence}

Relative genome divergence $\left(\mathrm{F}_{\mathrm{ST}}\right)$ and realized gene flow (probability of migration) is not

278 predicted by between the current geographic definitions of allopatry and parapatry: allopatry

279 between PNG and mainland Australia and either allopatry or parapatry within mainland

280 Australia, respectively (electronic supplementary material, figure 2, figure S3, top; Kruskal-

281 Wallis chi-squared $=2.58 \mathrm{e}-03, \mathrm{df}=1, \mathrm{p}=0.9595)$. Population pairs exhibit a range of divergence

282 levels regardless of whether the current barrier is terrestrial or marine. Divergence due to

283 isolation-by-distance is well supported by a correlation between the adjusted $\mathrm{F}_{\mathrm{ST}}$ and $\log$ of the

284 distance in $\mathrm{km}$ (Spearman's rank correlation: $\mathrm{rho}=0.427936, \mathrm{p}=0.004698)$. Correspondingly,

285 the probability of migration also decreases with increasing distance (Spearman's rank

286 correlation: rho $=-0.482106, \mathrm{p}=0.001225)$. Lastly, we compared the minumum landscape

287 resistance (ie. maximum range connectivity or maximum potential for gene flow corrected for

288 distance) between the three historic time points to $\mathrm{F}_{\mathrm{ST}}$ for each population pair. All comparisons

289 with PNG had the LGM as the period where PNG was connected with north Australia (18 pairs).

290 The timing of maximum connectivity within mainland Australia varied: eight pairs in present-

291 day, seven during the mid-Holocene, and nine in the LGM (electronic supplementary material,

292 table S7, figure S3). There is a positive correlation between landscape resistance and $\mathrm{F}_{\mathrm{ST}}$

293 (Spearman's rank correlation test, $\mathrm{S}=7048.6$, rho $=0.4288, \mathrm{p}=0.004601$ ), which translates to a

294 negative correlation between resistance and probability of gene flow (Spearman's rank

295 correlation test, $\mathrm{S}=18102$, rho $=-0.466829, \mathrm{p}=0.00183)$. In sum, whereas classification of

296 allopatry and parapatry based on predictions of current distribution do not predict divergence or

297 probability of gene flow, other factors such as geographic distance or past connectivity proves to

298 be more appropriate predictors. 

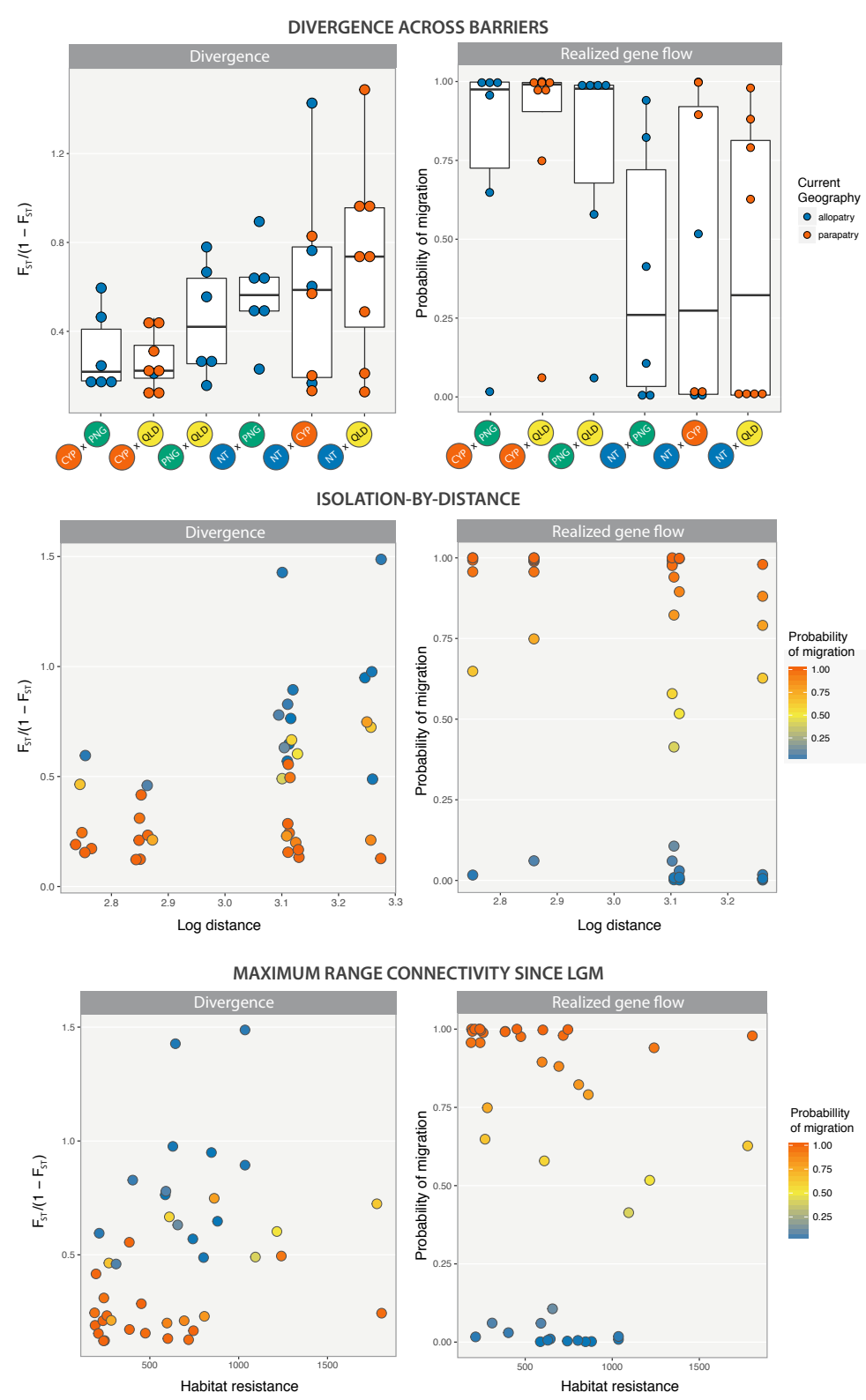

300 Figure 2. Top: Relative divergence through the barriers colored by whether or not population

301 pairs have disjunct ranges. Allopatry and parapatry are defined based on connectivity in the

302 species distribution models. The barriers are ordered based on increasing geographic distance

303 Middle: Adjusted $\mathrm{F}_{\mathrm{ST}}$ values plotted against log of distance to show isolation-by-distance. Color

304 gradient depicts the relative support for the models with gene flow. Points have been jittered by 
0.03 to display the number of points. Bottom: Divergence plotted against the highest connectivity across three time points (present, mid-Holocene, LGM).

Although the patterns described above also apply to the $\mathrm{Z}$ chromosome, the $\mathrm{Z}$

310 between the autosomal $\mathrm{F}_{\mathrm{ST}}$ value and $\mathrm{Z}$ chromosome Fst value increases as probability of gene

311 flow decreases (Spearman rank correlation: $r h o=-0.5504, p=1.58 \mathrm{e}-4$ ) but does not correlate

312 with current population connectivity (Kruskal-Wallis rank sum test: chi-squared $=0.12665$, $\mathrm{df}=$

$3131, \mathrm{p}=0.7219)$. This means that the amount of difference between autosomal and $\mathrm{Z}$ chromosome

314 divergence is a function of overall divergence rather than spatial context. The difference between

315 autosomal and $\mathrm{Z}$ chromosome values increases with increasing divergence for both relative $\left(\mathrm{F}_{\mathrm{ST}}\right)$

316 and absolute divergence $\left(\mathrm{D}_{\mathrm{XY}}\right)$, though absolute divergence far less so (Spearman rank

317 correlation $\mathrm{F}_{\mathrm{ST}}$ : rho $=0.715672, \mathrm{p}=1.001 \mathrm{e}-7$; Spearman rank correlation $\mathrm{D}_{\mathrm{XY}}:$ rho $=0.285595, \mathrm{p}$

$318=0.06674$; electronic supplementary material, figure S6). In this system, broad sampling of

319 population pairs reveal that difference between autosomal and $\mathrm{Z}$ chromosome divergence is

320 likely simply related to level of divergence rather than population connectivity.

321 Genome divergence and speciation

322 Designations of allopatry or parapatry in current distributions do not predict realized gene

323 flow, even for the less diverged populations. However, our data suggests that relative divergence

324 level influences realized gene flow throughout the entire range of divergence. We see a rapid

325 transition from high gene flow with low divergence to low gene flow with higher divergence

326 through a narrow range of divergence levels; similar to the model of snowballing during

327 speciation (figure 3). Support values for the different demographic models can be found in the 
electronic supplementary material table S10. A subset of simulations under the strict isolation model was always recovered to have low probability of migration regardless of $\mathrm{F}_{\mathrm{ST}}$ value, suggesting that the trajectory we see in our data is not likely due to artifacts in the model

331 selection (figure 3).
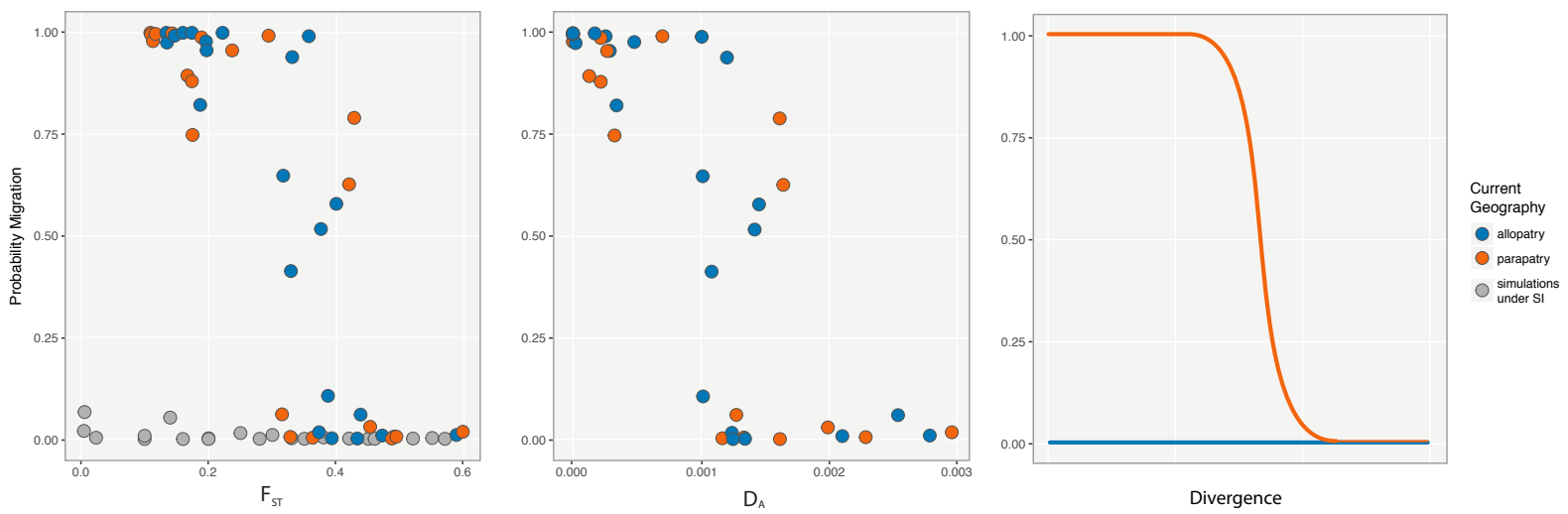

333 Figure 3. Probability of migration defined by the sum of ABC model supports for the 4 models

334 with a migration parameter (IM, IMhetM, IMhetN, IMhetNhetM) plotted against $\mathrm{F}_{\mathrm{ST}}$ and $\mathrm{D}_{\mathrm{A}}$.

335 The third panel is a plot of our expectation of the change in probability of migration under

336 allopatry and parapatry.

After comparing our data to models proposed by Yamaguchi and Iwasa [15], all

338 simulations retained by the $\mathrm{ABC}$ model selection were those simulated under the sigmoidal

339 function representative of a snowballing effect (electronic supplementary material, figure S2).

340 There is no support that our data follow any other proposed trajectories. The $\mathrm{F}_{\mathrm{ST}}$ range

341 corresponding to the tipping point spans $\sim 0.3-0.4$. The $\mathrm{D}_{\mathrm{A}}$ range corresponding to the tipping

342 point is $\sim 0.1-0.17 \%$. Species-specific points often span the entire range of the trajectory

343 (electronic supplementary material, figure S7). Plots for the Z chromosome, $\mathrm{D}_{\mathrm{XY}}$, and ND2

344 against probability of migration can be found in the electronic supplementary material, figure S8.

345 Our system provides additional empirical support for a snowballing pattern in speciation. 
Genome-wide divergence

The coefficient of variance describes the distribution of the individual $\mathrm{F}_{\mathrm{ST}}$ values across

348 the RAD loci. Higher coefficient of variance corresponds to a more skewed distribution - i.e. one

349 in which there is higher heterogeneity in levels of divergence across loci. At lower $\mathrm{F}_{\mathrm{ST}}$ values

350 (and high gene flow) there is a lower coefficient of variance as most values are close to zero. The

351 coefficient of variance increases with increasing $\mathrm{F}_{\mathrm{ST}}$ but peaks at intermediate levels of migration

352 and starts to decrease again when the support for migration decreases. The change in the

353 distribution of $F_{S T}$ values follow a predictable pattern with increasing divergence where there is

354 an initial skew from low to moderate divergence levels followed by a more uniform distribution

355 from moderate to high divergence levels (figure 4). This may be due to similarities in genome

356 architecture rather than differences in current geographic classification.

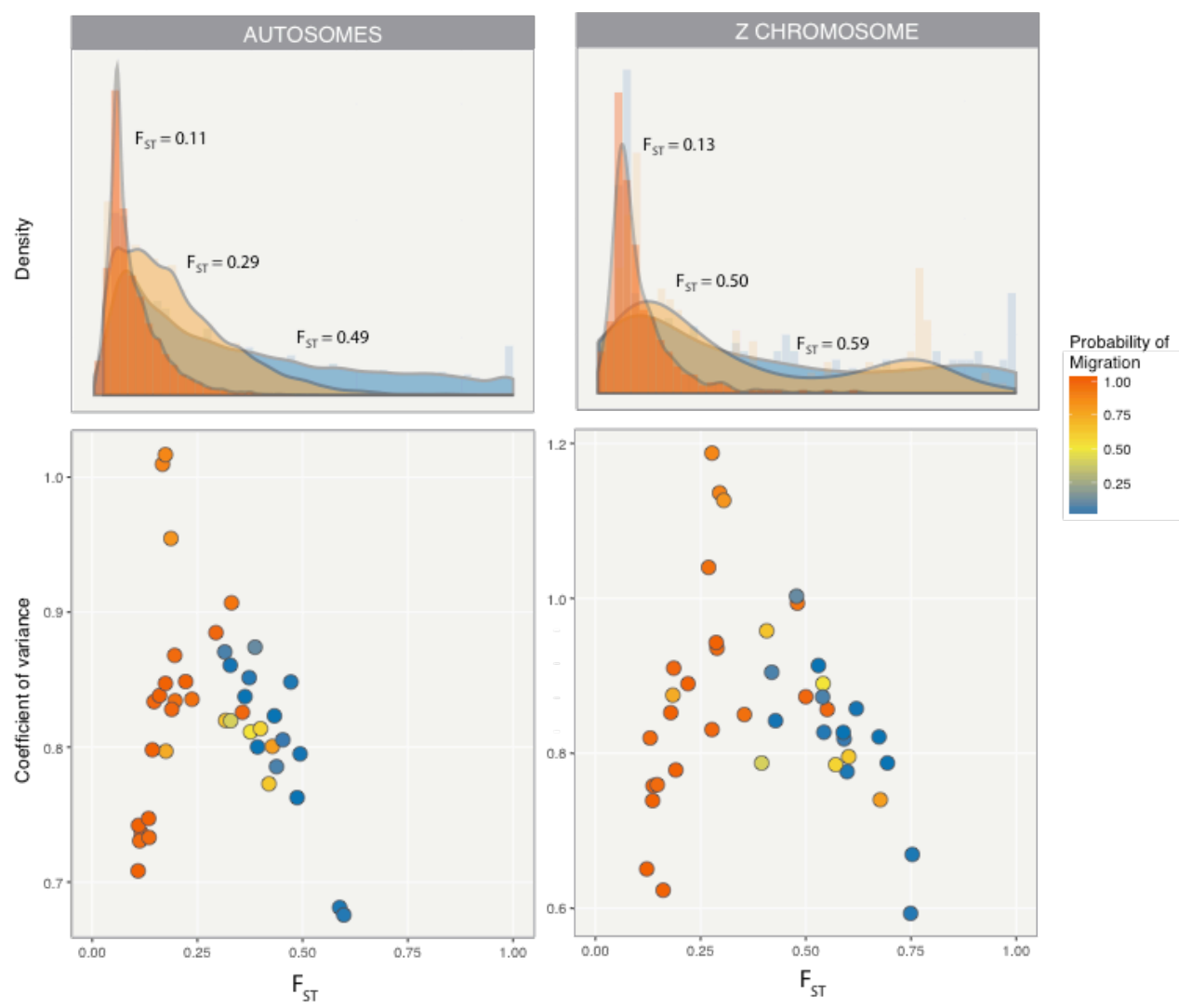


Figure 4. Top: Density of $\mathrm{F}_{\mathrm{ST}}$ distributions of the ddRAD loci with increasing global $\mathrm{F}_{\mathrm{ST}}$ and decreasing probability of migration. Three representative population pairs were chosen to represent different divergence levels. Low: Brown honeyeater NT \& QLD, Medium: Blue-faced honeyeater CYP \& QLD, high: White-throated honeyeater NT \& QLD. Bottom: Distribution of the skew of $\mathrm{F}_{\mathrm{ST}}$ distributions with increasing global $\mathrm{F}_{\mathrm{ST}}$ and decreasing probability of migration.

Discussion

Geographic mode of speciation is expected to influence the roles of gene flow, selection, and drift on divergence. However, our data suggests that although currently continuous geographic ranges should have higher potential for gene flow relative to discontinuous ranges, it does not necessarily translate to a higher probability of migration during divergence. The degree of connectivity between populations is dynamic through their evolutionary history and this results in reticulation of the genomes between those populations (figure 1, electronic supplementary material, figure S3)[59]. Our system supports the idea that current classifications of geography (allopatric, parapatric, and sympatric) are not reflective of gene flow during

372 population divergence [6,7]. It is possible that some currently allopatric populations have high

373 probability of gene flow reflecting past connectivity. Conversely, some currently parapatric

374 populations may have low probability of gene flow from incompatibilities accumulated during

375 past allopatry. Instead, there is support for geographic distance and minumum resistance being 376 negatively correlated with realized gene flow and positively correlated with divergence. Properly

377 classifying the geographic mode of speciation is particularly pertinent to a dynamic region like northern Australia and PNG. Despite this correlation with distance and historical connectivity,

379 population divergence and realized gene flow of these birds across various barriers in northern

380 Australia and Papua New Guinea span the range of the speciation continuum potentially due to 
381 lineage-specific biogeographic history or natural history (figure 3). Accumulating biogeographic

382 studies across suture zones or shared biogeographical barriers show similar dynamics of

383 population divergence in other avian systems as well as lizards and invertebrates [60-62].

384 Our data provides another empirical example in support for a snowballing pattern during

385 speciation. There exists a certain threshold of divergence where there is a rapid transition

386 between high and low gene flow likely resulting in speciation. Though populations at

387 intermediate stages are still observed, they persist at a narrow range of divergence levels. Our

388 data shows a similar rapid transition between the states of high and low likelihood for gene flow

389 (figure 3). This transition occurs at a narrow stage of nuclear and mitochondrial divergence; F $_{\text {ST }}$

$390 \sim 0.3-0.4, \mathrm{D}_{\mathrm{A}} \sim 0.1-0.17 \%$, and ND2 p-distances of $\sim 1-1.5 \%$. Using the substitution rate from

391 Pacheco et al. (2011), the ND2 p-distances would translate to 1.11 - 1.67 Mya. The range of $\mathrm{D}_{\mathrm{A}}$

392 where transition occurs is far lower to that found in Roux et al. ( $\sim 0.5-2 \%)$ which may be a

393 characteristic specific to avian speciation. Additional studies in other avian systems would help

394 determine if these patterns in divergence are robust.

Unlike the nuclear loci, there are outliers in the mitochondrial data where populations

396 with high mtDNA divergence would still have high likelihood of gene flow and low $\mathrm{F}_{\mathrm{ST}}$

397 (electronic supplementry material, figure S4). These populations are from the dusky myzomela

398 (CYP \& QLD, CYP \& PNG, and PNG \& QLD) and from the white-throated honeyeater (CYP \&

399 QLD). The dusky myzomela population pairs are part of the small subset which are all allopatric

400 on mainland Australia. One possible explanation for the white-throated honeyeater is that gene

401 flow after secondary contact has homogenized the nuclear genome but maintained the local ND2

402 haplotypes in accordance with Haldane's rule [63]. 
Although neither ecological selection nor incompatibility loci were explicitly tested, this

404

405

406

407

408

409

410

411

412

413

414

415

416

417

418

419

420

421

422

423

424

425

sigmoidal trajectory of speciation is consistent with the parapatric models (divergence-with-gene flow) proposed by Yamaguchi and Iwasa [15]. As suggested by the dynamic geographic history, population pairs likely experienced varying rates of gene flow through time $[8,59]$. On the other

hand, it has also been shown that a nonlinear accumulation of divergence can occur under certain neutral scenarios [20]. Ideally, estimates of population splitting time would inform us about the timing and duration of speciation and therefore the rate of accumulating divergence; however, we found that we could not use ddRAD to infer divergence times reliably [64].

Within the genome, there is variation of divergence across loci owing to various degrees of standing genetic variation and recombination [25-27]. The change in the distribution of $\mathrm{F}_{\mathrm{ST}}$ values across loci is a coarse estimate of the change in landscape of divergence through time.
Although the L-shape or the skew of $\mathrm{F}_{\mathrm{ST}}$ distributions between parapatrically diverging

populations have been attributed to a few loci under divergent selection with the rest

homogenized by gene flow [30,31], our broader sampling of population pairs show that the

change in $\mathrm{F}_{\mathrm{ST}}$ distribution follow a predictable pattern with increasing $\mathrm{F}_{\mathrm{ST}}$ or decreasing

probability of gene flow regardless of connectivity. It is also likely that this pattern of

accumulation of divergence is due to variation in nucleotide diversity across the loci $[20,25]$. The change in skew with increasing $\mathrm{F}_{\mathrm{ST}}$ could also be driven by linked selection instead of resistance to gene flow as recent studies have shown $[27,65]$. It would be important to complement this study with detailed studies across hybrid or suture zones to disentangle the role of gene flow and linked selection on divergence between hybridizing populations.

Finally, we note some important points for the study of the geographic mode of speciation. First, neither range overlap nor migration rate is static during speciation and it is 
more likely that populations experienced various degrees of geographic connectivity and gene

427 flow through time [59]. This is particularly pertinent for highly vagile taxa, like birds, in highly

428 dynamic geographic regions, like the Australopapuan region. Additionally, populations that are

429 currently allopatric due to vicariance could have experienced periods of reduced but ongoing

430 gene flow during the formation of the barrier [66]. Second, population differentiation would also

431 vary if a population has accumulated isolating mechanisms during allopatry and accelerated

432 divergence during secondary contact (alloparapatry) [2]. Depending on the particular

433 demographic history, isolation with continuous migration (primary divergence) can be difficult

434 to distinguish from gene flow after secondary contact from genetic data alone [24]. Third,

435 population divergence in allopatry may not necessarily translate to speciation. Seeing that most

436 species definitions rely on degree of reproductive isolation there are no consistent criteria to

437 differentiate discontinuous populations with no gene flow from allopatric species $[1,67,68]$.The

438 combination of reduced gene flow, either completely or partially, and genetic drift may result in

439 population divergence but other factors likely play a more important role in speciation.

\section{Conclusion}

$441 \quad$ Our comparative study of divergence of bird populations through the speciation process

442 highlights the dynamics of geographic history and their influence on divergence. Further, it

443 provides additional support for a snowballing pattern in speciation, and it characterizes broad

444 patterns of genomic divergence through time. The divergence that we discuss in this paper is

445 presumed to be neutral and therefore would benefit from a replicate study looking at divergence

446 in coding regions to observe whether different marker types have different trajectories in the

447 same populations. The results of this broad study also clearly describe the pattern of

448 accumulation of divergence which lend support to the emerging relevance of linked selection in 
genome divergence. Comparative studies, particularly with multiple species in a shared geographic region, help elucidate patterns of genome divergence during speciation. To fully

451 comprehend the patterns of neutral and adaptive genomic divergence, we need to sample broadly

452 both phylogenetically and geographically thus affecting shared patterns in speciation

453 differentiated from the exceptions.

454 Author contributions

455 JVP conceived ideas and designed the project, collected the data, performed the analyses, and led

456 the writing of the manuscript. LJ and CM provided ideas during the analysis and writing of the

457 manuscript

458 Acknowledgments

459 We would like to thank Ian J. Mason and Alex Drew for greatly appreciated help with collecting 460 samples in the field, Alexander Xue for assistance with demographic analyses, Matteo Fumigalli

461 for assistance with ngsTools, and Daniel Rosauer for assistance with species distribution

462 modelling. We would also like to thank Sonal Singhal, Sally Potter, and Daniel R. Wait for

463 helpful discussions and comments on the manuscript. Lastly all data collection was carried out in 464 Australian National University's Biomolecular Research Facility and most analyses were carried 465 out in the ABC Bioinformatics Development Cluster.

$466 \quad$ Funding

467 This research was funded by BirdLife Stuart Leslie Bird Research Award 2015.

$468 \quad$ References

469 1. Mayr E. 1942 Systematics and the Origin of Species, from the Viewpoint of a Zoologist. $470 \quad$ Harvard University Press.

471 2. Coyne JA, Orr HA, Others. 2004 Speciation. Sinauer Associates Sunderland, MA.

472 3. White MJD. 1978 Modes of speciation. San Francisco: WH Freeman 455p.-Illus., maps, 
chrom. nos.. General (KR, 197800185).

474 4. Mayr E. 1963 Animal species and evolution. Animal species and evolution.

475 5. Gavrilets S. 2003 Perspective: models of speciation: what have we learned in 40 years?

$476 \quad$ Evolution 57, 2197-2215.

477

6. Butlin RK, Galindo J, Grahame JW. 2008 Sympatric, parapatric or allopatric: the most important way to classify speciation? Philos. Trans. R. Soc. Lond. B Biol. Sci. 363, 29973007.

7. Losos JB, Glor RE. 2003 Phylogenetic comparative methods and the geography of speciation. Trends Ecol. Evol. 18, 220-227.

8. Hofreiter M, Stewart J. 2009 Ecological change, range fluctuations and population dynamics during the Pleistocene. Curr. Biol. 19, R584-94.

9. Seehausen O et al. 2014 Genomics and the origin of species. Nat. Rev. Genet. 15, 176-192.

10. Orr HA. 1995 The population genetics of speciation: the evolution of hybrid incompatibilities. Genetics

11. Welch JJ. 2004 Accumulating Dobzhansky-Muller incompatibilities: reconciling theory and data. Evolution 58, 1145-1156.

12. Orr HA, Turelli M. 2001 The evolution of postzygotic isolation: accumulating DobzhanskyMuller incompatibilities. Evolution 55, 1085-1094.

13. Gavrilets S. 2000 Waiting time to parapatric speciation. Proc. Biol. Sci. 267, 2483-2492.

14. Gavrilets S. 2014 Models of Speciation: Where Are We Now? J. Hered. 105, 743-755.

15. Yamaguchi R, Iwasa Y. 2017 A tipping point in parapatric speciation. J. Theor. Biol. 421, 81-92.

16. Yamaguchi R, Iwasa Y. 2013 First passage time to allopatric speciation. Interface Focus 3, 20130026.

17. Via S. 2012 Divergence hitchhiking and the spread of genomic isolation during ecological speciation-with-gene-flow. Philos. Trans. R. Soc. Lond. B Biol. Sci. 367, 451-460.

18. Feder JL, Egan SP, Nosil P. 2012 The genomics of speciation-with-gene-flow. Trends Genet. 28, 342-350.

19. Feder JL, Nosil P, Wacholder AC, Egan SP, Berlocher SH, Flaxman SM. 2014 GenomeWide Congealing and Rapid Transitions across the Speciation Continuum during Speciation with Gene Flow. J. Hered. 105, 810-820.

20. Southcott L, Kronforst MR. In press. A neutral view of the evolving genomic architecture 
of speciation. Ecol. Evol. (doi:10.1002/ece3.3190)

21. Matute DR, Butler IA, Turissini DA, Coyne JA. 2010 A Test of the Snowball Theory for the Rate of Evolution of Hybrid Incompatibilities. Science 329, 1518-1521.

22. Moyle LC, Nakazato T. 2010 Hybrid incompatibility 'snowballs' between Solanum species. Science 329, 1521-1523.

23. Riesch $\mathrm{R}$ et al. 2017 Transitions between phases of genomic differentiation during stickinsect speciation. Nature Ecology \& Evolution 1, 0082.

24. Roux C, Fraïsse C, Romiguier J, Anciaux Y, Galtier N, Bierne N. 2016 Shedding Light on the Grey Zone of Speciation along a Continuum of Genomic Divergence. PLoS Biol. 14, e2000234.

25. Cruickshank TE, Hahn MW. 2014 Reanalysis suggests that genomic islands of speciation are due to reduced diversity, not reduced gene flow. Mol. Ecol. 23, 3133-3157.

26. Ravinet M, Faria R, Butlin RK, Galindo J, Bierne N, Rafajlović M, Noor MAF, Mehlig B, Westram AM. 2017 Interpreting the genomic landscape of speciation: a road map for finding barriers to gene flow. J. Evol. Biol. 30, 1450-1477.

27. Burri R et al. 2015 Linked selection and recombination rate variation drive the evolution of the genomic landscape of differentiation across the speciation continuum of Ficedula flycatchers. Genome Res. 25, 1656-1665.

28. Ellegren $\mathrm{H}$ et al. 2012 The genomic landscape of species divergence in Ficedula flycatchers. Nature 491, 756-760.

29. Martinsen GD, Whitham TG, Turek RJ, Keim P. 2001 Hybrid populations selectively filter gene introgression between species. Evolution 55, 1325-1335.

30. Martin SH et al. 2013 Genome-wide evidence for speciation with gene flow in Heliconius butterflies. Genome Res. (doi:10.1101/gr.159426.113)

31. Nosil P, Gompert Z, Farkas TE, Comeault AA, Feder JL, Buerkle CA, Parchman TL. 2012 Genomic consequences of multiple speciation processes in a stick insect. Proc. Biol. Sci. 279, 5058-5065.

32. Edwards RD, Crisp MD, Cook DH, Cook LG. 2017 Congruent biogeographical disjunctions at a continent-wide scale: Quantifying and clarifying the role of biogeographic barriers in the Australian tropics. PLoS One 12, e0174812.

33. Cracraft J. 1986 Origin and evolution of continental biotas: speciation and historical congurence within the Australian avifauna. Evolution 40, 977-996.

34. Bowman DMJS et al. 2010 Biogeography of the Australian monsoon tropics. J. Biogeogr. 37, 201-216. 
35. Lambeck K, Chappell J. 2001 Sea Level Change Through the Last Glacial Cycle. Science 292, 679-686.

36. Baldassarre DT, White TA, Karubian J, Webster MS. 2014 Genomic and morphological analysis of a semipermeable avian hybrid zone suggests asymmetrical introgression of a sexual signal. Evolution 68, 2644-2657.

37. Catullo RA, Lanfear R, Doughty P, Keogh JS. 2014 The biogeographical boundaries of northern Australia: evidence from ecological niche models and a multi-locus phylogeny of Uperoleia toadlets (Anura: Myobatrachidae). J. Biogeogr. 41, 659-672.

38. Edwards SV, Potter S, Schmitt CJ. 2016 Reticulation, divergence, and the phylogeographyphylogenetics continuum. Proceedings of the

39. Kearns AM, Joseph L, Omland KE, Cook LG. 2011 Testing the effect of transient PlioPleistocene barriers in monsoonal Australo-Papua: did mangrove habitats maintain genetic connectivity in the Black Butcherbird? Mol. Ecol. 20, 5042-5059.

40. Peñalba JV, Mason IJ, Schodde R, Moritz C, Joseph L. In press. Characterizing divergence through three adjacent Australian avian transition zones. J. Biogeogr. (doi:10.1111/jbi.13048)

41. R. Schodde, Mason IJ. 1999 The Directory of Australian Birds. Passerines. CSIRO Publishing: Melbourne

42. Ford J. 1987 Hybrid Zones in Australian Birds. Еmu 87, 158-178.

43. Sorenson MD, Ast JC, Dimcheff DE, Yuri T, Mindell DP. 1999 Primers for a PCR-based approach to mitochondrial genome sequencing in birds and other vertebrates. Mol. Phylogenet. Evol. 12, 105-114.

44. Peterson BK, Weber JN, Kay EH, Fisher HS, Hoekstra HE. 2012 Double digest RADseq: an inexpensive method for de novo SNP discovery and genotyping in model and non-model species. PLoS One 7, e37135.

45. Eaton DAR. 2014 PyRAD: assembly of de novo RADseq loci for phylogenetic analyses. Bioinformatics 30, 1844-1849.

46. Langmead B, Salzberg SL. 2012 Fast gapped-read alignment with Bowtie 2. Nat. Methods 9, 357-359.

47. Korneliussen TS, Albrechtsen A, Nielsen R. 2014 ANGSD: Analysis of Next Generation Sequencing Data. BMC Bioinformatics 15, 356.

48. Fumagalli M, Vieira FG, Korneliussen TS, Linderoth T, Huerta-Sánchez E, Albrechtsen A, Nielsen R. 2013 Quantifying population genetic differentiation from next-generation sequencing data. Genetics 195, 979-992. 
49. Vieira FG, Lassalle F, Korneliussen TS, Fumagalli M. 2016 Improving the estimation of genetic distances from Next-Generation Sequencing data. Biol. J. Linn. Soc. Lond. 117, 139-149.

50. Huson DH. 1998 SplitsTree: analyzing and visualizing evolutionary data. Bioinformatics 14, 68-73.

51. Reynolds J, Weir BS, Cockerham CC. 1983 Estimation of the coancestry coefficient: basis for a short-term genetic distance. Genetics 105, 767-779.

52. Weir BS, Cockerham CC. 1984 ESTIMATING F-STATISTICS FOR THE ANALYSIS OF POPULATION STRUCTURE. Evolution 38, 1358-1370.

53. Hartl DL, Clark AG, Clark AG. 1997 Principles of population genetics. Sinauer associates Sunderland.

54. Korneliussen TS, Moltke I, Albrechtsen A, Nielsen R. 2013 Calculation of Tajima's D and other neutrality test statistics from low depth next-generation sequencing data. $B M C$

Bioinformatics 14, 289.

55. Paradis E, Claude J, Strimmer K. 2004 APE: Analyses of Phylogenetics and Evolution in R language. Bioinformatics 20, 289-290.

56. Csilléry K, François O, Blum MGB. 2012 abc: an R package for approximate Bayesian computation (ABC). Methods Ecol. Evol. 3, 475-479.

57. Hijmans RJ, Elith J. 2013 Species distribution modeling with R. R package version 0. 8-11

58. van Etten J. 2017 R Package gdistance: Distances and Routes on Geographical Grids. Journal of Statistical Software, Articles 76, 1-21.

59. Rheindt FE, Edwards SV. 2011 Genetic introgression: an integral but neglected component of speciation in birds. Auk 128, 620-632

60. Singhal S, Bi K. 2017 History cleans up messes: the impact of time in driving divergence and introgression in a tropical suture zone. Evolution (doi:10.1111/evo.13278)

61. Winger BM, Bates JM. 2015 The tempo of trait divergence in geographic isolation: avian speciation across the Marañon Valley of Peru. Evolution 69, 772-787.

62. Whinnett A, Zimmermann M, Willmott KR, Herrera N, Mallarino R, Simpson F, Joron M, Lamas G, Mallet J. 2005 Strikingly variable divergence times inferred across an Amazonian butterfly 'suture zone'. Proc. Biol. Sci. 272, 2525-2533.

63. Price TD, Bouvier MM. 2002 The evolution of F1 postzygotic incompatibilities in birds. Evolution 56, 2083-2089.

64. Shafer ABA, Peart CR, Tusso S, Maayan I, Brelsford A, Wheat CW, Wolf JBW. 2016 
Bioinformatic processing of RAD-seq data dramatically impacts downstream population genetic inference. Methods Ecol. Evol. (doi:10.1111/2041-210X.12700)

608

609

610

611

612

613

614

615

616

617

65. Wang J, Street NR, Scofield DG, Ingvarsson PK. 2016 Variation in Linked Selection and Recombination Drive Genomic Divergence during Allopatric Speciation of European and American Aspens. Mol. Biol. Evol. 33, 1754-1767.

66. Yang M, He Z, Shi S, Wu C-I. 2017 Can genomic data alone tell us whether speciation happened with gene flow? Mol. Ecol. 26, 2845-2849.

67. Harvey MG, Seeholzer GF, Smith BT, Rabosky DL, Cuervo AM, Brumfield RT. 2017 Positive association between population genetic differentiation and speciation rates in New World birds. Proc. Natl. Acad. Sci. U. S. A. (doi:10.1073/pnas.1617397114)

68. Sukumaran J, Knowles LL. 2017 Multispecies coalescent delimits structure, not species. Proc. Natl. Acad. Sci. U. S. A. 114, 1607-1612. 